\title{
Doença de Chagas aguda no estado do Pará, Brasil: série histórica de aspectos clínicos e epidemiológico em três municípios, no período de 2007 a 2015
}

\section{Acute Chagas disease in Pará State, Brazil: historical series of clinical and epidemiological aspects in three municipalities, from 2007 to 2015}

Andrezza Ozela de Vilhena' (iD), Waltair Maria Martins Pereira² (iD), Silvio Silva de Oliveira² (iD, Paulo Fernando Lauria Fonseca $^{3}$ (D), Milene Silveira Ferreira ${ }^{4}$ iD, Tatyellen Natasha da Costa Oliveira ${ }^{1,4}$ iD, Marcos Adami ${ }^{5}$ (D), Patricia Danielle Lima de Lima' ${ }^{\text {(D) }}$

\author{
' Universidade do Estado do Pará, Belém, Pará, Brasil \\ 2 Universidade Federal do Pará, Belém, Pará, Brasil \\ ${ }^{3}$ Centro Universitário do Estado do Pará, Belém, Pará, Brasil \\ ${ }^{4}$ Instituto Evandro Chagas, Ananindeua, Pará, Brasil \\ ${ }^{5}$ Instituto Nacional de Pesquisas Espaciais, Belém, Pará, Brasil
}

\begin{abstract}
RESUMO
OBJETIVO: Analisar o perfil clínico e epidemiológico da doença de Chagas aguda nos municípios de Belém, Abaetetuba e Breves, estado do Pará, Brasil, de 2007 a 2015. MATERIAIS E MÉTODOS: Foram analisados 696 casos, a partir de dados coletados do Sistema de Informação de Agravos de Notificação, e aplicados testes estatísticos, por meio do software BioEstat. RESULTADOS: Dos 696 investigados, 35,63\% eram provenientes de Abaetetuba, 40,66\% de Belém e 23,71\% de Breves. A faixa etária de 30 a 59 anos foi a mais prevalente, sendo 35,89\% em Abaetetuba e $53,71 \%$ em Belém; diferentemente, em Breves, 32,73\% tinham entre 0 e 14 anos de idade. Os homens acometidos representaram $51,61 \%$ em Abaetetuba, 49,47\% em Belém e 56,36\% em Breves. A área urbana registrou 56,45\% dos casos em Abaetetuba e $96,11 \%$ em Belém; e, na zona rural de Breves, residiam 66,06\% dos casos. A taxa de letalidade foi de 1,49\%. A via de transmissão mais frequente foi a oral (82,33\%). A febre e a astenia estiveram presentes em acima de $75 \%$ dos registros. A curva epidêmica da sazonalidade da doença foi marcante entre os meses de julho e novembro, e a incidência foi mais expressiva em Breves, sendo observadas duas ondas epidêmicas: uma em 2009 (27,98/100.000 habitantes) e outra em 2015 (63,38/100.000 habitantes). CONCLUSÃO: A relevância epidemiológica da doença de Chagas aguda e sua importância para a saúde pública são justificadas pelo potencial epidêmico do parasita, o que exige a organização de serviços de saúde, atividades de prevenção e controle da doença.
\end{abstract}

Palavras-chave: Doença de Chagas; Epidemiologia Descritiva; Doenças Negligenciadas; Indicador de Saúde.

\begin{abstract}
OBJECTIVE: To analyze the clinical and epidemiological profile of acute Chagas disease in the municipalities of Belém, Abaetetuba, and Breves, Pará State, Brazil, from 2007 to 2015. MATERIALS AND METHODS: A total of 696 cases were analyzed, using data from the Information on Notifiable Diseases, and statistical tests were applied using the BioEstat software. RESULTS: Of the 696 investigated, 35.63\% were from Abaetetuba, 40.66\% from Belém and 23.71\% from Breves. The most prevalent age group was $30-59$ years, being $35.89 \%$ in Abaetetuba and $53.71 \%$ in Belém; in contrast, in Breves, 32.73\% were between 0 and 14 years old. Affected men represented $51.61 \%$ in Abaetetuba, 49.47\% in Belém, and $56.36 \%$ in Breves. The urban area registered $56.45 \%$ of the cases in Abaetetuba and $96.11 \%$ in Belém; and in the rural area of Breves $66.06 \%$ of cases. The mortality rate was $1.49 \%$. The oral transmission (82.33\%) was predominant. Fever and asthenia were present in over $75 \%$ of records. The epidemic curve of seasonality was higher between July and November, and the incidence was more expressive in Breves, with two epidemic waves: one in 2009 (27.98/100,000 inhabitants) and another in 2015 (63.38/100,000 inhabitants). CONCLUSION: The epidemiological relevance of acute Chagas disease and its importance for public health are justified by the epidemic potential of the parasite, which requires the organization of health services, activities for the prevention and control of the disease.
\end{abstract}

Keywords: Chagas Disease; Descriptive Epidemiology; Neglected Diseases; Health Indicator.

\author{
Correspondência / Correspondence: \\ Andrezza Ozela de Vilhena \\ Trav. Nove de Janeiro, 471. Bairro: Umarizal. CEP: 66060-370 - Belém, Pará, Brasil - Tel.: +55 (91) 98848-4773 \\ E-mail: aozelav@gmail.com.br
}




\section{INTRODUÇÃO}

Após mais de um século da descoberta da doença de Chagas (DC) por Carlos Chagas (1909), essa patologia ainda representa uma das mais importantes doenças negligenciadas no mundo'. A DC é uma antropozoonose causada pelo protozoário flagelado Trypanosoma cruzi, que é transmitido ao homem pelas fezes dos insetos vetores, principalmente dos gêneros Triatoma, Panstrongylus e Rhodnius ${ }^{2}$. A transmissão também ocorre pelas vias transfusional, transplacentária e $\mathrm{oral}^{3}$.

Estima-se que 65 milhões de indivíduos vivem sob o risco da infecção em países endêmicos das Américas, sendo 12.000 infectados anualmente ${ }^{4}$. Entre os anos de 2003 a 2018, foram notificados 4.556 casos de DC aguda (DCA) no Brasil. Até o ano de 2007, a maioria ocorreu na Região Nordeste; contudo, a partir da constatação da transmissão oral $^{3}$, esse cenário mudou, passando a Região Norte a apresentar a maior incidência ${ }^{5}$.

A DCA é considerada endêmica na Região Amazônica, com frequentes surtos epidêmicos entre membros de uma mesma família, ocorrendo em áreas urbanas e rurais $6,7,8$. No período de 2006 a 2014, - estado do Pará registrou 884 casos da doença e, desses, 25,7\% (227) eram da Região Metropolitana de Belém e 21,1\% (187) de Abaetetuba; 26,9\% (238) foram registrados no arquipélago do Marajó, dos quais 44,5\% (106) eram de Breves ${ }^{4}$.

No Pará, novos casos agudos vêm sendo notificados de forma crescente, razão pela qual os serviços de vigilância epidemiológica estão cada vez mais em alerta, mantendo contínua atenção em populações de maior risco e, sobretudo, estimulando o envolvimento dos profissionais que atuam na atenção a saúde, para que novos casos sejam prontamente diagnosticados e tratados, buscando, assim, a melhoria desse cenário que tem desfechos muitas vezes incapacitantes se não houver um atendimento eficaz e imediato?.

A DC apresenta curso clínico bifásico, composto por uma fase aguda, por vezes não identificada, evoluindo para a fase crônica. É considerada aguda quando o indivíduo apresenta febre prolongada (por mais de sete dias), acompanhada ou não de edema de face ou de membros, exantema, adenomegalia, hepatomegalia, esplenomegalia, cardiopatia aguda (taquicardia, sinais de insuficiência cardíaca), manifestações hemorrágicas, icterícia, sinal de Romaña ou chagoma de inoculação ${ }^{10}$.

O potencial epidêmico da DC no Pará justifica a importância de estudos epidemiológicos de base populacional que possam auxiliar no planejamento estratégico de atividades de vigilância a saúde visando - controle da doença. Nesse cenário, objetivou-se descrever o perfil clínico-epidemiológico de portadores da DCA nos municípios de Abaetetuba, Belém e Breves, no estado do Pará.

\section{MATERIAIS E MÉTODOS}

Trata-se de um estudo transversal retrospectivo, baseado em dados secundários, obtidos do Sistema de Informação de Agravos de Notificação (SINAN) do Ministério da Saúde do Brasil (MS) ${ }^{1}$. Foram incluídos 696 casos de DCA, com confirmação laboratorial e/ou epidemiológica, que residiam nos municípios de Abaetetuba, Belém e Breves, no período de 2007 a 2015.

O município de Abaetetuba situa-se no nordeste do

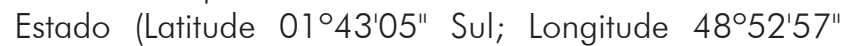
Oeste) e possuía \pm 153.380 habitantes (hab.) no ano de 2017; Belém, capital do Estado (Latitude: $1^{\circ} 27^{\prime} 18^{\prime \prime}$ Sul; Longitude: 48 30' 9" Oeste), tinha uma população estimada de \pm 1.452.275 hab. no mesmo ano; e Breves, considerado o maior arquipélago do Marajó, possuía \pm 99.896 hab. também naquele ano ${ }^{12}$ (Figura 1).

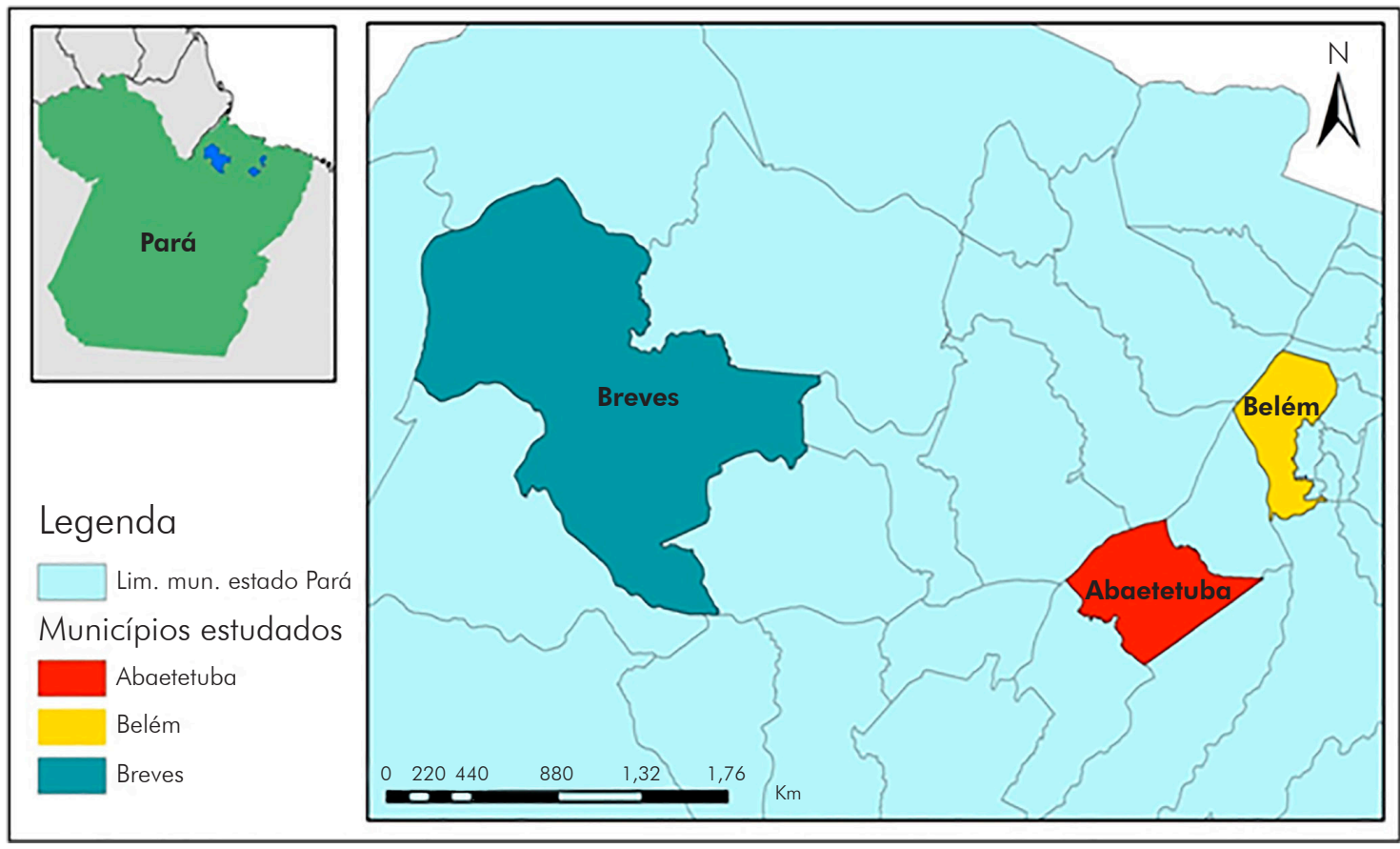

Fonte: Instituto Brasileiro de Geografia e Estatística, 2015

Figura 1 - Localização geográfica dos municípios de Abaetetuba, Belém e Breves, estado do Pará, Brasil 
Foram analisadas as seguintes variáveis: i) perfil epidemiológico: município e zona de residência, gênero, faixa etária, raça/cor da pele, escolaridade, mês e ano do diagnóstico/notificação, critério de confirmação, evolução do caso, modo provável de infecção, tratamento para assintomáticos e sintomáticos e diagnóstico laboratorial (gota espessa, Strout/micro-hematócrito e Quantitative Buffy Coat); ii) perfil clínico: edema de face/membros, meningoencefalite, poliadenopatia, febre persistente, hepatomegalia, sinais de insuficiência cardíaca congestiva (ICC), astenia, esplenomegalia e chagoma de inoculação/sinal de Romaña.

As taxas brutas de incidência foram calculadas e padronizadas pela população mundial e apresentadas por 100.000 hab. Para o cálculo da sazonalidade, os casos notificados e confirmados foram contados e agrupados pelo mês da data dos primeiros sintomas, considerando os 12 meses dos anos da série estudada, utilizando a função lógica do Microsoft Excel. A sazonalidade, na série temporal, foi suavizada pela média móvel simples. Para a análise estatística inferencial, aplicou-se o teste qui-quadrado de aderência de proporções iguais e esperadas para a análise da distribuição de frequências utilizando o software BioEstat v5.013. Os dados foram apresentados em tabelas e gráficos produzidos no Microsoft Excel 2013.

\section{RESULTADOS}

Dos 696 casos de DCA investigados, 40,66\% (283/696) residiam em Belém, 35,63\% (248/696) em Abaetetuba e 23,71\% (165/696) em Breves. O gênero masculino foi predominante em 51,61\% (128/248) dos casos de Abaetetuba e em 56,36\% (93/165) dos casos de Breves; em Belém, as mulheres foram as mais afetadas, representando 50,53\% (143/283) dos casos. A faixa etária de 30 a 59 anos foi a mais acometida, sendo 35,89\% (89/248) em Abaetetuba e $53,71 \%$ (152/283) em Belém; o que diferiu em Breves, onde $32,73 \%$ (54/165) tinham entre de 0 a 14 anos de idade. A maioria dos investigados declararam ser pardos, sendo 78,23\% (194/248) de Abaetetuba, $68,90 \%$ (195/283) de Belém e 89,70\% (148/165) de Breves. A baixa escolaridade prevaleceu entre os casos: $42,74 \%(106 / 248)$ e $63,64 \%$ (105/165) dos investigados possuíam Ensino Fundamental incompleto em Abaetetuba e Breves, respectivamente, e 17,69\% (50/283) cursou até o Ensino Médio em Belém. Destaca-se o alto percentual de sub-registro da escolaridade de 34,63\% (98/283) em Belém. Na área urbana, residiam 56,45\% (140/248) e 96,11\% (272/283) dos casos de Abaetetuba e Belém, respectivamente; em Breves, 66,06\% (109/165) dos casos ocorreram na zona rural (Tabela 1).

diagnóstico laboratorial foi realizado em 94,35\% (234/248) dos casos em Abaetetuba, 97,88\% (277/283) em Belém e 88,48\% (146/165) em Breves ( $<<0,0001)$. A maioria dos investigados sobreviveu à infecção e recebeu alta para acompanhamento posterior ao tratamento específico. Desses, 97,17\% (241/248) eram de Abaetetuba, 92,94\% (263/283) de Belém e $98,18 \%(162 / 165)$ de Breves $(p<0,0001)$. Os três municípios notificaram óbitos, sendo 1,21\% (3/248) em Abaetetuba, 2,12\% (6/283) em Belém e 0,61\% (1/165) em Breves. A taxa de letalidade foi de 1,43\% nos três municípios. A infecção pela via oral ocorreu em 85,08\% (21 1/248) dos casos de Abaetetuba, 71,38\% (202/283) em Belém e 96,97\% (160/165) em Breves ( $p<0,0001)$. tratamento específico para os assintomáticos foi realizado em 98,39\% (244/248), 96,11\% (272/283) e 93,33\% (154/165) dos casos de Abaetetuba, Belém e Breves, respectivamente. Entre os sintomáticos, receberam tratamento 60,08\% (149/248) dos casos de Abaetetuba, 6,36\% (18/283) de Belém e 49,09\% $(81 / 165)$ de Breves. Quanto ao diagnóstico laboratorial, a gota espessa foi realizada em 66,13\% (164/248) dos casos em Abaetetuba e 71,52\% (118/165) em Breves. Diferentemente, o micro-hematócrito foi o exame mais realizado em Belém, em 45,58\% (129/283) dos casos (Tabela 2).

Apresentaram sintomas 94,35\% (234/248) dos casos de Abaetetuba, 89,40\% (253/283) de Belém e 95,75\% (158/165) de Breves. A febre persistiu em $91,93 \%$ (228/248) dos investigados de Abaetetuba, 93,64\% (265/283) de Belém e 93,33\% (154/165) de Breves. Astenia esteve presente com frequência acima de $75 \%$ dos casos nos três municípios. Edema de face/membros, meningoencefalite e hepatomegalia foram evidenciados em 42,05\% (119/283), 1,06\% $(3 / 283)$ e $7,77 \%$ (22/283) dos casos de Belém, respectivamente. Esplenomegalia foi observada em 4,84\% (12/248) nos casos de Abaetetuba, 4,59\% (13/283) em Belém e 13,33\% (22/165) em Breves; e taquicardia persistente/arritmias foram notados em 20,16\% (50/248) dos casos em Abaetetuba, 13,78\% (39/283) em Belém e 16,97\% (28/165) em Breves. Poliadenopatia foi observada em 1,61\% (4/248) dos casos de Abaetetuba; e a presença de chagoma ocorreu em 2,42\% (4/164) dos casos de Breve (Tabela 3).

A distribuição mensal de casos de DCA registrou uma curva epidêmica de sazonalidade. Foi observado um discreto aumento no mês de fevereiro, em Belém (15 casos) e Abaetetuba (10 casos), seguindo uma tendência decrescente, e posterior aumento de grande amplitude a partir do mês de julho, nos três municípios estudados. Para Abaetetuba, a alça ascendente foi contínua e registrou um pico epidêmico em novembro (81 casos), decrescendo acentuadamente em dezembro (30 casos). Para Belém, a alça ascendente iniciou em abril (um caso) e registrou pico epidêmico em outubro (70 casos), regredindo posteriormente até dezembro (28 casos). Para Breves, as notificações começaram a partir de julho (sem registro de casos), seguindo posteriormente para dois picos epidêmicos, o maior em setembro (39 casos) e o menor em novembro (28 casos) (Figura 2).

Abaetetuba apresentou três picos epidêmicos nos anos de 2009 (77,24/100.000 hab.), 2012 (44,29/100.000 hab.) e 2014 (22,86/100.000 hab.), com tendência linear em discreto decréscimo $\left(R^{2}=0,0267\right)$, o que pode ser observado na figura 3 (linha pontilhada). Belém apresentou a menor taxa de incidência entre os municípios estudados e apresentou tendência linear estável $\left(R^{2}=0,0011\right)$. Em Breves, foram evidenciadas duas ondas epidêmicas, uma em $2009(27,98 / 100.000$ hab.) e outra em 2015 $(63,38 / 100.000$ hab.), registrando alta tendência linear crescente $\left(R^{2}=0,2504\right)$ mostrada também na figura 3 (linha pontilhada). 
Tabela 1 - Perfil sociodemográfico dos casos de DCA nos municípios de Abaetetuba, Belém e Breves, estado do Pará, Brasil, de 2007 a 2015

\begin{tabular}{|c|c|c|c|c|c|c|}
\hline \multirow{2}{*}{ Variáveis } & \multicolumn{2}{|c|}{ Abaetetuba } & \multicolumn{2}{|c|}{ Belém } & \multicolumn{2}{|c|}{ Breves } \\
\hline & $N=248$ & $\%$ & $N=283$ & $\%$ & $N=165$ & $\%$ \\
\hline \multicolumn{7}{|l|}{ Gênero } \\
\hline Masculino & 128 & 51,61 & 140 & 49,47 & 93 & 56,36 \\
\hline Feminino & 120 & 48,39 & 143 & 50,53 & 72 & 43,64 \\
\hline \multicolumn{7}{|l|}{ Faixa etária } \\
\hline $0-14$ & 64 & 25,80 & 26 & 9,19 & 54 & 32,73 \\
\hline $15-29$ & 65 & 26,21 & 66 & 23,32 & 46 & 27,88 \\
\hline 30-59 & 89 & 35,89 & 152 & 53,71 & 48 & 29,09 \\
\hline$\geq 60$ & 30 & 12,10 & 39 & 13,78 & 17 & 10,30 \\
\hline \multicolumn{7}{|l|}{ Cor da pele } \\
\hline Branca & 41 & 16,53 & 24 & 8,48 & 9 & 5,45 \\
\hline Preta & 6 & 2,42 & 2 & 0,71 & 3 & 1,82 \\
\hline Amarela & - & - & - & - & 1 & 0,61 \\
\hline Parda & 194 & 78,23 & 195 & 68,90 & 148 & 89,70 \\
\hline Indígena & - & - & - & - & 2 & 1,21 \\
\hline Ignorado & 4 & 1,61 & 53 & 18,73 & 2 & 1,21 \\
\hline Sem informação & 3 & 1,21 & 9 & 3,18 & - & - \\
\hline \multicolumn{7}{|l|}{ Escolaridade } \\
\hline Sem escolaridade & 8 & 3,23 & 2 & 0,71 & 22 & 13,33 \\
\hline Ensino Fundamental incompleto & 106 & 42,74 & 47 & 16,61 & 105 & 63,64 \\
\hline Ensino Fundamental completo & 24 & 9,68 & 46 & 16,25 & 6 & 3,64 \\
\hline Ensino Médio & 50 & 20,16 & 50 & 17,67 & 9 & 5,45 \\
\hline Ensino Superior & 4 & 1,61 & 17 & 6,01 & - & - \\
\hline lgnorado & 9 & 3,63 & 98 & 34,63 & 3 & 1,82 \\
\hline Não se aplica & 29 & 11,69 & 8 & 2,82 & 16 & 9,70 \\
\hline Sem informação & 18 & 7,26 & 15 & 5,30 & 4 & 2,42 \\
\hline \multicolumn{7}{|l|}{ Zona de residência } \\
\hline Urbana & 140 & 56,45 & 272 & 96,11 & 53 & 32,12 \\
\hline Periurbana & 1 & 0,40 & 1 & 0,35 & 2 & 1,21 \\
\hline Rural & 96 & 38,71 & 7 & 2,48 & 109 & 66,06 \\
\hline Ignorado & - & - & 1 & 0,35 & - & - \\
\hline Sem informação & 11 & 4,44 & 2 & 0,71 & 1 & 0,61 \\
\hline
\end{tabular}


Tabela 2 - Perfil clínico e epidemiológico dos casos de DCA nos municípios de Abaetetuba, Belém e Breves, estado do Pará, Brasil, de 2007 a 2015

\begin{tabular}{|c|c|c|c|c|c|c|}
\hline \multirow{2}{*}{ Variáveis } & \multicolumn{2}{|c|}{ Abaetetuba } & \multicolumn{2}{|c|}{ Belém } & \multicolumn{2}{|c|}{ Breves } \\
\hline & $N=248$ & $\%$ & $N=283$ & $\%$ & $N=165$ & $\%$ \\
\hline \multicolumn{7}{|l|}{ Critério de confirmação } \\
\hline Clínico e epidemiológico & 14 & 5,65 & 4 & 1,42 & 18 & 10,91 \\
\hline Clínico & - & - & 1 & 0,35 & 1 & 0,61 \\
\hline Laboratorial & 234 & $94,35^{*}$ & 277 & $97,88^{*}$ & 146 & $88,48^{*}$ \\
\hline Sem informação & - & - & 1 & 0,35 & - & - \\
\hline \multicolumn{7}{|l|}{ Evolução do caso } \\
\hline Vivo & 241 & $97,17^{*}$ & 263 & $92,94^{*}$ & 162 & $98,18^{*}$ \\
\hline Óbito por DCA & 3 & 1,21 & 6 & 2,12 & 1 & 0,61 \\
\hline Óbito por outra causa & - & - & 1 & 0,35 & - & - \\
\hline Ignorado & 2 & 0,81 & 6 & 2,12 & - & - \\
\hline Sem informação & 2 & 0,81 & 7 & 2,47 & 2 & 1,21 \\
\hline \multicolumn{7}{|l|}{ Modo provável de infecção } \\
\hline Transfusional & - & - & 4 & 1,42 & - & - \\
\hline Vetorial & 27 & 10,89 & 3 & 1,06 & 4 & 2,42 \\
\hline Vertical & 1 & 0,40 & - & - & - & - \\
\hline Oral & 211 & $85,08^{*}$ & 202 & $71,38^{*}$ & 160 & $96,97^{*}$ \\
\hline lgnorado & 9 & 3,63 & 67 & 23,67 & 1 & 0,61 \\
\hline Sem informação & - & - & 7 & 2,47 & - & - \\
\hline \multicolumn{7}{|l|}{ Tratamento específico } \\
\hline Sim & 244 & 98,39 & 272 & 96,11 & 154 & 93,33 \\
\hline Não & 3 & 1,21 & 6 & 2,12 & 3 & 1,82 \\
\hline Sem informação & 1 & 0,40 & 5 & 1,77 & 8 & 4,85 \\
\hline \multicolumn{7}{|l|}{ Tratamento sintomático } \\
\hline Sim & 149 & 60,08 & 18 & 6,36 & 81 & 49,09 \\
\hline Não & 91 & 36,69 & 77 & 27,21 & 67 & 40,60 \\
\hline Ignorado & 0 & 0,00 & 12 & 4,24 & 1 & 0,61 \\
\hline Sem informação & 8 & 3,23 & 176 & 62,19 & 16 & 9,70 \\
\hline \multicolumn{7}{|c|}{ Exame a fresco/gota esp./esfregaço } \\
\hline Positivo & 164 & 66,13 & 65 & 22,97 & 118 & 71,52 \\
\hline Negativo & 75 & 30,24 & 51 & 18,02 & 26 & 15,76 \\
\hline Não realizado & 6 & 2,42 & 122 & 43,11 & 17 & 10,30 \\
\hline Sem informação & 3 & 1,21 & 45 & 15,90 & 4 & 2,42 \\
\hline \multicolumn{7}{|c|}{ Strout/micro-hematócrito/QBC } \\
\hline Positivo & 2 & 0,81 & 129 & 45,58 & 5 & 3,03 \\
\hline Negativo & 39 & 15,72 & 86 & 30,39 & 36 & 21,82 \\
\hline Não realizado & 201 & 81,05 & 28 & 9,90 & 118 & 71,52 \\
\hline Sem informação & 6 & 2,42 & 40 & 14,13 & 6 & 3,63 \\
\hline
\end{tabular}


Tabela 3 - Perfil clínico dos casos de DCA nos municípios de Abaetetuba, Belém e Breves, estado do Pará, Brasil, de 2007 a 2015

\begin{tabular}{|c|c|c|c|c|c|c|}
\hline \multirow{2}{*}{ Variáveis } & \multicolumn{2}{|c|}{ Abaetetuba } & \multicolumn{2}{|c|}{ Belém } & \multicolumn{2}{|c|}{ Breves } \\
\hline & $N=248$ & $\%$ & $N=283$ & $\%$ & $N=165$ & $\%$ \\
\hline \multicolumn{7}{|l|}{ Assintomático } \\
\hline Sim & 14 & 5,65 & 8 & 2,83 & 6 & 3,64 \\
\hline Não & 234 & 94,35 & 253 & 89,40 & 158 & 95,75 \\
\hline lgnorado & - & - & 3 & 1,06 & - & - \\
\hline Sem informação & - & - & 19 & 6,71 & 1 & 0,61 \\
\hline \multicolumn{7}{|l|}{ Edema de face/membros } \\
\hline Sim & 67 & 27,02 & 119 & 42,05 & 34 & 20,61 \\
\hline Não & 166 & 66,93 & 141 & 49,82 & 124 & 75,15 \\
\hline Ignorado & - & - & 5 & 1,77 & - & - \\
\hline Sem informação & 15 & 6,05 & 18 & 6,36 & 7 & 4,24 \\
\hline \multicolumn{7}{|l|}{ Meningoencefalite } \\
\hline Sim & 2 & 0,81 & 3 & 1,06 & - & - \\
\hline Não & 228 & 91,93 & 222 & 78,45 & 157 & 95,15 \\
\hline lgnorado & 3 & 1,21 & 30 & 10,60 & 1 & 0,61 \\
\hline Sem informação & 15 & 6,05 & 28 & 9,89 & 7 & 4,24 \\
\hline \multicolumn{7}{|l|}{ Poliadenopatia } \\
\hline Sim & 4 & 1,61 & 3 & 1,06 & 3 & 1,82 \\
\hline Não & 216 & 87,10 & 218 & 77,03 & 154 & 93,33 \\
\hline Ignorado & 13 & 5,24 & 32 & 11,31 & 1 & 0,61 \\
\hline Sem informação & 15 & 6,05 & 30 & 10,60 & 7 & 4,24 \\
\hline \multicolumn{7}{|l|}{ Febre persistente } \\
\hline Sim & 228 & 91,93 & 265 & 93,64 & 154 & 93,33 \\
\hline Não & 6 & 2,42 & 10 & 3,53 & 3 & 1,82 \\
\hline lgnorado & - & - & - & - & - & - \\
\hline Sem informação & 14 & 5,65 & 8 & 2,83 & 8 & 4,85 \\
\hline \multicolumn{7}{|l|}{ Hepatomegalia } \\
\hline Sim & 24 & 9,68 & 22 & 7,77 & 23 & 13,94 \\
\hline Não & 200 & 80,65 & 220 & 77,74 & 135 & 81,82 \\
\hline Ignorado & 8 & 3,22 & 13 & 4,59 & - & - \\
\hline Sem informação & 16 & 6,45 & 28 & 9,90 & 7 & 4,24 \\
\hline \multicolumn{7}{|l|}{ Sinais de ICC } \\
\hline Sim & 6 & 2,42 & 16 & 5,65 & 5 & 3,03 \\
\hline Não & 225 & 90,72 & 206 & 72,79 & 152 & 92,12 \\
\hline lgnorado & 3 & 1,21 & 33 & 11,66 & 1 & 0,61 \\
\hline Sem informação & 14 & 5,65 & 28 & 9,90 & 7 & 4,24 \\
\hline \multicolumn{7}{|c|}{ Taquicardia persistente/arritmias } \\
\hline Sim & 50 & 20,16 & 39 & 13,78 & 28 & 16,97 \\
\hline Não & 171 & 68,95 & 194 & 68,55 & 131 & 79,39 \\
\hline lgnorado & 12 & 4,84 & 26 & 9,19 & - & - \\
\hline Sem informação & 15 & 6,05 & 24 & 8,48 & 6 & 3,64 \\
\hline \multicolumn{7}{|l|}{ Astenia } \\
\hline Sim & 187 & 75,40 & 223 & 78,80 & 127 & 76,97 \\
\hline Não & 45 & 18,14 & 44 & 15,55 & 30 & 18,18 \\
\hline Ignorado & 2 & 0,81 & 1 & 0,35 & - & - \\
\hline Sem informação & 14 & 5,65 & 15 & 5,30 & 8 & 4,85 \\
\hline \multicolumn{7}{|l|}{ Esplenomegalia } \\
\hline Sim & 12 & 4,84 & 13 & 4,59 & 22 & 13,33 \\
\hline Não & 211 & 85,08 & 229 & 80,92 & 136 & 82,43 \\
\hline Ignorado & 9 & 3,63 & 12 & 4,24 & - & - \\
\hline Sem informação & 16 & 6,45 & 29 & 10,25 & 7 & 4,24 \\
\hline \multicolumn{7}{|c|}{ Chagoma de inoculação/sinal de Romaña } \\
\hline Sim & 2 & 0,81 & 2 & 0,70 & 4 & 2,42 \\
\hline Não & 228 & 91,93 & 244 & 86,22 & 152 & 92,12 \\
\hline Ignorado & 3 & 1,21 & 8 & 2,83 & - & - \\
\hline Sem informação & 15 & 6,05 & 29 & 10,25 & 9 & 5,46 \\
\hline
\end{tabular}

Ignorado: o investigado não sabia ou não podia informar; Sem informação: dado não registrado. Sinal convencional utilizado: - Dado numérico igual a zero, não resultante de arredondamento. 


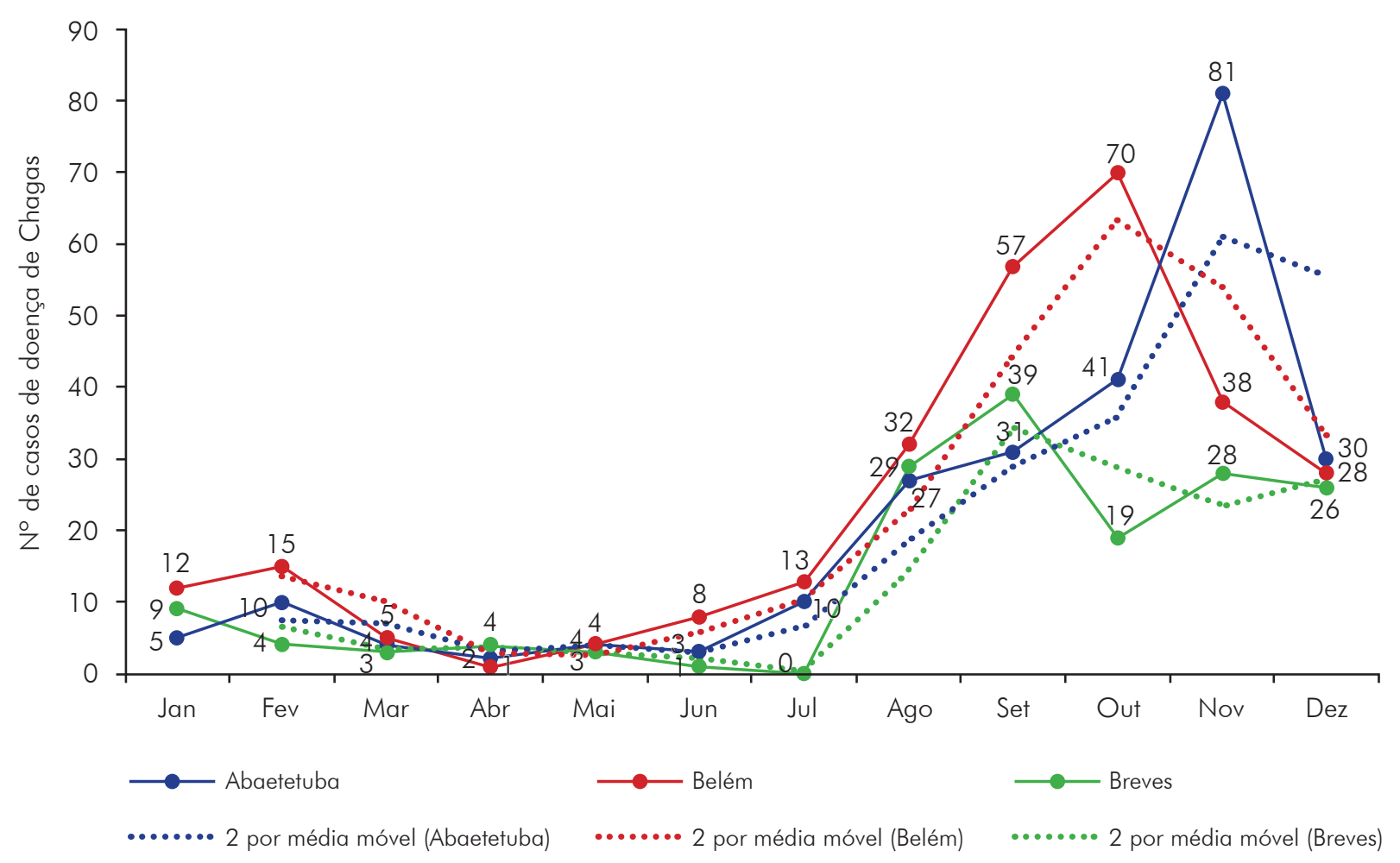

Fonte: SINAN/MS; SESPA 2017.

Figura 2 - Distribuição mensal dos casos acumulados de DCA e média móvel nos municípios de Abaetetuba, Belém e Breves, estado do Pará, Brasil, de 2007 a 2015

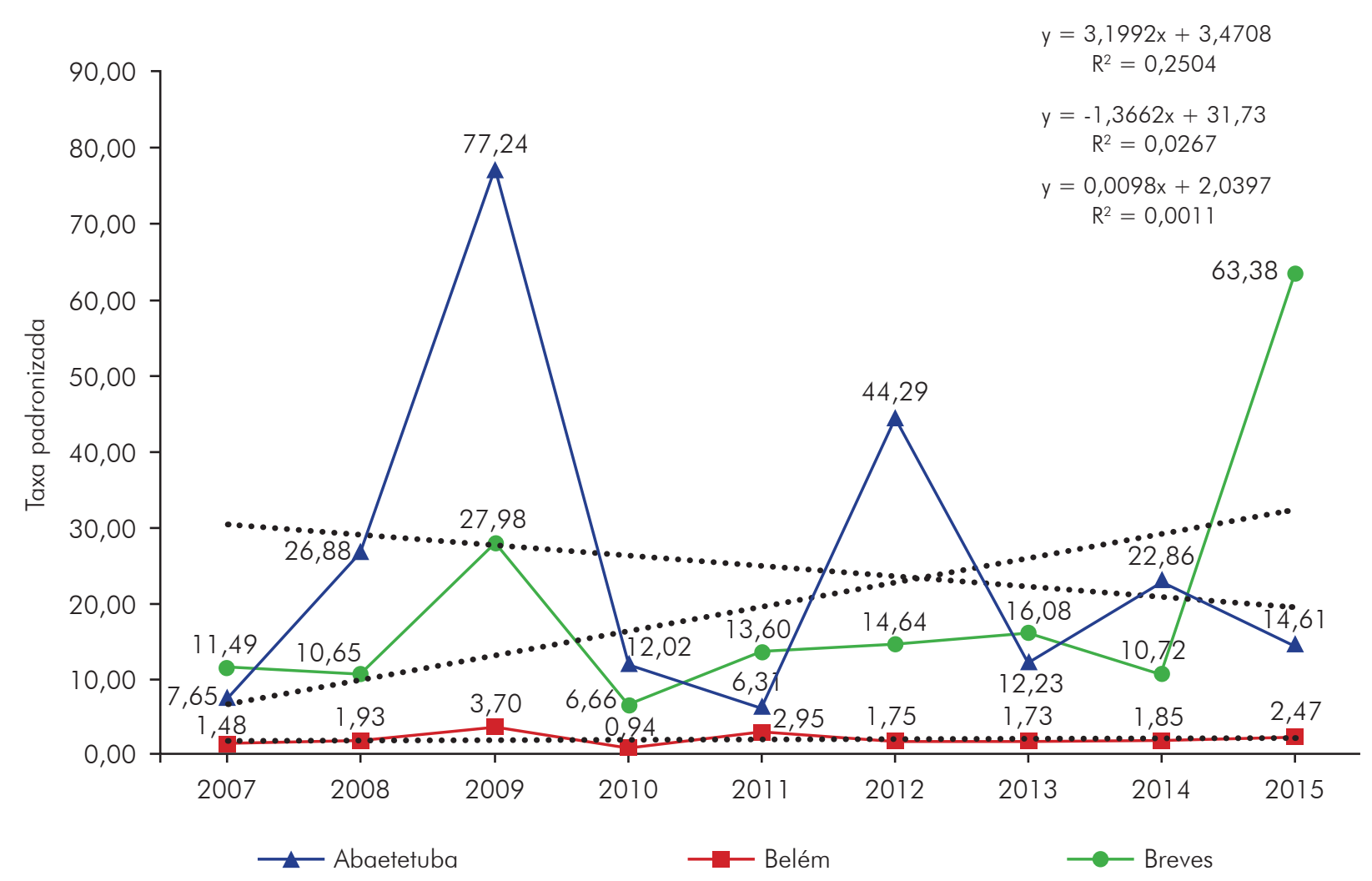

Fonte: SINAN/MS; SESPA 2017

Figura 3 - Taxa de incidência padronizada de DCA nos municípios de Abaetetuba, Belém e Breves, estado do Pará, Brasil, de 2007 a 2015 


\section{DISCUSSÃO}

As ações de controle vetorial e a intensa vigilância na triagem de doadores de sangue e órgãos, realizada a partir de 1975, reduziram substancialmente o número de casos de DCA no Brasil ${ }^{14}$. No entanto, a partir da década de 1990, a Região Norte, área anteriormente considerada não endêmica, adquiriu importância em saúde pública, em virtude das inusitadas condições epidemiológicas de transmissão concorrentes (oral, indireta), que desencadearam a retomada da notificação obrigatória de casos agudos em $2000^{15}$. A obrigatoriedade da notificação trouxe à tona um problema de saúde pública negligenciado há décadas na Amazônia. De 2007 a 2019, o Pará registrou uma média de 187,38 casos de DCA. Dos 154 surtos registrados no Brasil, no período de 2007 a 2016, 132 ocorreram no Pará, distribuídos em 20 dos seus municípios ${ }^{15}$. Barcarena, município localizado no nordeste do Estado, teve a maior prevalência da doença no Brasil, no período de 2007 a $2014^{16}$. Ao relatar a ocorrência de 283/696 casos de DCA em Belém, 248/696 em Abaetetuba e 165/696 em Breves, a presente série histórica reforça esse contexto epidemiológico e a inquestionável magnitude da doença na região.

Neste estudo, a DCA não mostrou preferência por gênero e faixa etária. Em Abaetetuba e Breves, os homens representaram a maioria dos casos e, em Belém, as mulheres foram as mais acometidas. Os adultos (30-59 anos de idade) foram os mais infectados pelo T. cruzi em Abaetetuba e Belém, e os jovens (0-14 anos), em Breves. Acredita-se que a exposição às diversas rotas de transmissão é o que provavelmente determina a infecção ${ }^{17}$. Isso é bem exemplificado com a falta de consonância quanto à distribuição do sexo e a idade dos infectados documentados no país $s^{6,76,17}$. A predominância da cor parda declarada resulta por ocasião do Censo de 2010, quando $76,5 \%$ das pessoas se autodeclararam negros e/ou pardos ${ }^{18}$, o que justifica a prevalência dos pardos entre os infectados nos três municípios estudados e corrobora um estudo realizado no município de Barcarena ${ }^{16}$. $\bigcirc$ perfil sociodemográfico dos casos de DCA em Abaetetuba, Belém e Breves se assemelha ao observado no país. Dos 3.060 casos de DCA registrados no período de 2007 a 2019, os homens representaram 53,56\% dos casos, a média de idade foi de 32 anos (DP $\pm 20,15)$ e $77,81 \%$ se declararam pardos ${ }^{15}$.

Os resultados deste estudo demonstraram que tanto - meio rural quanto o urbano apresentam impactos frente à DCA. Os casos foram notificados em grandes centros urbanos, como Belém, capital do Estado (40,66\%), e em cidades menores ou mesmo em áreas rurais, como observado em Abaetetuba (35,63\%) e Breves (23,71\%), respectivamente. A emergência e reemergência da DCA em áreas urbanas e rurais resulta da combinação de diversos fatores como: correntes migratórias, desequilíbrio ecológico, aspectos socioculturais e político-econômicos ${ }^{19,20}$.
O desmatamento das áreas rurais e o crescimento desordenado das cidades, devido à migração populacional de áreas rurais para as urbanas, têm facilitado a urbanização do vetor ${ }^{9,21}$. A maior proporção de casos de DCA registrados na região urbana de Abaetetuba e Belém é justificada por essa expressiva migração populacional. Simões et al. ${ }^{22}$ estimaram que 500.000 pessoas infectadas por T. cruzi deslocaram-se para as grandes cidades durante as últimas três décadas. Ao se fixar nos centros urbanos, a população vive em condições de extrema pobreza e saneamento básico precário, o que propicia o aumento do risco de infecção, o crescimento e a permanência de doenças negligenciadas ${ }^{23}$.

No Pará, o desmatamento recorrente há décadas contribui para a diminuição do habitat de animais silvestres, que atuam como reservatório natural do T. cruzi. $\bigcirc$ desequilíbrio ecológico causa a aproximação do homem com os reservatórios, facilitando a transmissão do protozoário pelo vetor e a contaminação de alimentos (açaí) que não são processados obedecendo às boas práticas de manipulação dos mesmos ${ }^{24}$. Essa cadeia de transmissão da doença foi demonstrada nos resultados do presente estudo. A via oral foi o modo provável de infecção da maioria dos investigados $(p<0,0001)$. Breves, o município com a menor taxa de desenvolvimento entre os municípios estudados e com a maior cobertura vegetal, apresentou a maior taxa de infecção pela via oral (96,97\%), seguido por Abaetetuba (85,08\%) e Belém (71,38\%). No entanto, o registro da infecção por doação de sangue/órgãos infectados somente em Belém (1,42\%) mostrou a necessidade da implementação e/ou fortalecimento de uma hemovigilância integrada ${ }^{15}$.

A falta de acesso a bens e serviços pela precariedade social e econômica foi retratada nos dados deste estudo. Nos três municípios estudados a maioria dos infectados por T. cruzi tinha baixa escolaridade (Ensino Fundamental ou Médio). Em Belém, 6,01\% (17/283) dos acometidos cursaram - Ensino Superior; em Abaetetuba, esses indivíduos representaram 1,61\% (4/248); e, em Breves, esse dado não foi registrado, talvez devido à dificuldade ao acesso à educação superior. Tais dados demonstram que indivíduos com maior grau de escolaridade têm maior possibilidade de acesso a informações sobre a doença, o que leva à mudança de hábitos, diminuição da contaminação e consequente atenuação do número de casos, conforme relatado por Sanmartino e Crocco $^{25}$.

A taxa de letalidade da doença em Abaetetuba $(1,21 \%)$ e em Breves $(0,61 \%)$ foi semelhante à taxa de letalidade anual no Brasil (1,54\%) e no Pará (1,40\%) para o período de 2007 a $2019^{15}$. Em Belém, a taxa de letalidade foi superior (2,12\%) quando comparada aos demais municípios avaliados, ao estado e ao país. Isso decorre devido a Belém atuar como o maior polo de atendimento hospitalar para casos de DCA no Pará e, por vezes, registrar o contingente de casos que evoluem a óbito oriundos de outros municípios. A 
implementação da assistência à pessoa com DCA nos municípios mais longínquos e vulneráveis da Amazônia é de extrema urgência. Tal serviço viabilizaria uma intervenção preventiva, ou seja, as ações ocorreriam antes do surgimento das complicações da doença aguda. A oportunidade de diagnóstico precoce seguramente é outro ponto de extrema importância, pois interfere no prognóstico dos casos e, de forma expressiva, na letalidade da doença ${ }^{4}$. A falta de investimentos para o diagnóstico da DCA nos municípios estudados foi demonstrada quando o esfregaço (baixo custo) foi o método mais utilizado para o diagnóstico da doença em Abaetetuba (66,13\%) e Breves (71,52\%), e o micro-hematócrito, em Belém $(45,58 \%)$. Cabe lembrar, que não existem critérios clínicos que possibilitem definir, com exatidão, a cura de pacientes com DCA, o que reforça não somente a importância do diagnóstico precoce, mas também o diagnóstico (sorológico) para o monitoramento da doença ${ }^{26}$.

A apresentação clínica foi acompanhada com maior frequência por síndrome febril e astenia. Estudos anteriores apontaram que a febre foi a manifestação clínica predominante na quase totalidade dos $\operatorname{casos}^{27,28,29}$. $\bigcirc$ edema, embora em menores proporções nos indivíduos infectados dos municípios de Abaetetuba $(27,02 \%)$ e Breves $(20,61 \%)$, foi observado em $42,05 \%$ dos casos de Belém, concordando com pesquisa realizada em Manaus, que identificou $31 \%$ dos casos com edema de membros inferiores e $34,5 \%$ com edema de face ${ }^{29}$. Os baixos percentuais de ocorrência de chagoma de inoculação/sinal de Romanã registrados nos municípios analisados resultam da diminuição das taxas de infeção pela via vetorial. Mais de $93 \%$ dos casos notificados nos três municípios estudados receberam tratamento específico (indivíduos assintomáticos) assegurado pelo SUS, com - objetivo de reduzir a casuística por meio da cura da infecção, da prevenção de lesões orgânicas ou ainda da evolução das mesmas ${ }^{30}$; entretanto, não há indícios de acompanhamento dos casos após a alta medicamentosa, por pelo menos cinco anos, para verificar a evolução, considerando a possibilidade de cronificação da doença.

A análise do comportamento temporal da DCA configura um importante mecanismo para a compreensão do perfil epidemiológico dessa enfermidade, que pode servir de base para melhorar as medidas de educação em saúde e atividades de proteção específica, objetivando a redução da ocorrência de casos. A distribuição mensal de casos de DCA para os três municípios registrou curva epidêmica de sazonalidade, com um aumento discreto no segundo mês do ano para Abaetetuba e Belém; e posterior aumento na frequência de casos de julho a outubro, período em que são registrados os menores índices pluviométricos, os quais favorecem a maior mobilidade de triatomíneos e o que pode facilitar a contaminação do ambiente e de frutos alimentícios com fezes infectadas. Esse período também coincide com a safra do açaí, o que aumenta o consumo do suco e o risco de infecção ${ }^{31}$.

A maior taxa de incidência da doença foi observada em Breves, por apresentar razão de crescimento de tendência $\left(R^{2}=0,2504\right)$ e por ter apresentado dois picos epidêmicos, um em 2009 e outro em 2015. Em Abaetetuba, a incidência da doença teve tendência linear em discreto decréscimo $\left(R^{2}=0,0267\right)$, ainda que tenham sido evidenciados três picos epidêmicos em 2009, 2012 e 2014. Belém chamou a atenção ao apresentar tendência linear estável $\left(R^{2}=0,0011\right)$ e a menor taxa de incidência entre os municípios estudados, apesar do pico epidêmico registrado no mês de outubro, que cursou até dezembro. A diferença da incidência da doença em Abaetetuba, Belém e Breves está intimamente relacionada ao grau de desenvolvimento socioeconômico e às políticas públicas de controle e combate da doença em cada localidade. Historicamente, os povos e comunidades tradicionais (agricultores familiares, trabalhadores rurais, populações ribeirinhas, comunidades quilombolas e indígenas) que enfrentam situações de desigualdades, violência e violação de direitos são os mais afetados pela doença ${ }^{15}$.

\section{CONCLUSÃO}

presente estudo descreveu o perfil epidemiológico dos casos de DCA notificados nos municípios de Abaetetuba, Belém e Breves, sendo indivíduos de ambos os sexos, jovens e adultos e residentes de áreas rurais e urbanas. A vulnerabilidade da população negligenciada mostrou-se o fator preponderante para o aumento do risco de infecção, seguido da destruição do meio ambiente, que favorece a transmissão do T. cruzi pelo vetor e a contaminação de alimentos (açaí), e da fragilidade ou ausência de uma vigilância sanitária integrada. A curva epidêmica de sazonalidade foi marcante entre os meses de agosto e novembro; e o município de Breves apresentou a maior taxa de incidência da doença, seguido de Abaetetuba e Belém. Considerando as relações das variáveis estudadas, os dados obtidos foram satisfatórios para a construção do cenário epidemiológico, o qual pode auxiliar os gestores em saúde com informações voltadas para a vigilância contínua e sistemática, que garanta o acesso universal como direito.

\section{CONFLITOS DE INTERESSES}

Os autores declaram não possui conflitos de interesses.

\section{CONTRIBUIÇÃO DOS AUTORES}

Todos os autores contribuíram de forma igualitária na concepção, desenvolvimento e elaboração do manuscrito referente a pesquisa em tela. 


\section{REFERÊNCIAS}

1 Dias JCP, Coura JR. Clínica e terapêutica da doença de Chagas: uma abordagem prática para - clínico geral. Rio de Janeiro: Editora Fiocruz; 1997. Capítulo 3, Epidemiologia; p. 33-66.

2 Galvão C, organizador. Vetores da doença de Chagas no Brasil. Curitiba: Sociedade Brasileira de Zoologia; 2014. 289 p. Série Zoologia: guias e manuais de identificação.

3 Dias JCP, Borges Dias R. Aspectos sociais, econômicos e culturais da doença de Chagas. Cienc Cult. 1979;31:105-24.

4 Ministério da Saúde (BR). Secretaria de Vigilância em Saúde. Departamento de Vigilância de Doenças e Agravos não Transmissíveis e Promoção da Saúde. Saúde Brasil 2017: uma análise da situação de saúde e os desafios para o alcance dos objetivos de desenvolvimento sustentável [Internet]. Brasília: Ministério da Saúde; 2018 [citado 2019 jun 17]. 426 p. Disponível em: http://tabnet. datasus.gov.br/cgi/sinasc/saude_brasil_2017 analise_situacao_saude_desafios_objetivos_ desenvolvimento_sustetantavel.pdf.

5 Lima MM, Alves RV, Costa JNG, Silva RA, Palmeira SL, Costa VM, et al. Doença de Chagas. Bol Epidemiol [Internet]. 2019 set [citado 2017 nov 16];50(n.esp.):16-8. Disponível em: https:// ameci.org.br/wp-content/uploads/2019/09/ boletim-especial-21 ago 19-web.pdf.

6 Coura JR. The main sceneries of Chagas disease transmission. The vectors, blood and oral transmissions - a comprehensive review. Mem Inst Oswaldo Cruz. 2015 May; 1 10(3):277-82.

7 Barbosa MGV, Ferreira JMBB, Arcanjo ARL, Santana RAG, Magalhães LKC, Magalhães LKC, et al. Chagas disease in the State of Amazonas: history, epidemiological evolution, risks of endemicity and future perspectives. Rev Soc Bras Med Trop. 2015 Jun;48(Suppl 1):27-33.

8 Beltrão HBM, Cerroni MP, Freitas DRC, Pinto AYN, Valente VC, Valente SA, et al. Investigation of two outbreaks of suspected oral transmission of acute Chagas disease in the Amazon region, Para State, Brazil, in 2007. Trop Doct. 2009 Oct;39(4):231-2.

9 Souza DSM, Monteiro MRCC. Manual de recomendações para diagnóstico, tratamento e seguimento ambulatorial de portadores de doença de Chagas. Belém: As Autoras; 2013. 50 p.

10 Ministério da Saúde (BR). Secretaria de Vigilância em Saúde. Coordenação-Geral de Desenvolvimento da Epidemiologia em Serviços. Guia de vigilância em saúde: volume único. 2. ed. Brasília: Ministério da Saúde; 2017. p. 441-61.

11 Secretaria de Estado da Saúde do Pará. Diretoria de Vigilância em Saúde. Departamento de Epidemiologia. Sistema de agravos de notificação. Belém: SESPA; 2018.
12 Fundação Amazônia de Amparo a Estudos e Pesquisas. Anuário Estatístico do Pará 2018: População total e estimativas populacionais, Pará e municípios - 2013 a 2017 [Internet]. Belém: FAPESPA; 2018 [citado 2002 jul 23]. Disponível em: http://www.fapespa.pa.gov.br/sistemas/anuario 2018/tabelas/demografia/tab_1.1_populacao_ total_e_estimativas_populacionais_para_e_ municipios_2013_a_2017.htm.

13 Ayres M, Ayres Jr M, Ayres DL, Santos AAS. BioEstat 5.0: aplicações estatísticas nas áreas das ciências biológicas e médicas. Belém: Sociedade Mamirauá; 2007. 364 p.

14 Dias JCP, Cláudio LDG, Lima MM, Albajar-Viñas P, Silva RA, Alves RV, et al. Mudanças no paradigma da conduta clínica e terapêutica da doença de Chagas: avanços e perspectivas na busca da integralidade da saúde. Epidemiol Serv Saude. 2016 jun;25(n. esp.):87-90.

15 Ministério da Saúde (BR). Secretaria de Vigilância em Saúde. Doença de Chagas: 14 de abril - Dia Mundial. Bol Epidemiol. 2020 abr;51(n.esp.): 1-43.

16 Sousa Jr AS, Palácios VRCM, Miranda CS, Costa RJF, Catete CP, Chagasteles EJ, et al. Análise espaço-temporal da doença de Chagas e seus fatores de risco ambientais e demográficos no município de Barcarena, Pará, Brasil. Rev Bras Epidemiol. 2017 out-dez;20(4):742-55.

17 Coura JR, Borges-Pereira J. Chagas disease: 100 years after its discovery. A systemic review. Acta Trop. 2010 Jul-Aug; 1 15(1-2):5-13.

18 Instituto Brasileiro de Geografia e Estatística. Censo Demográfico de 2010 [Internet]. Rio de Janeiro: Instituto Brasileiro de Geografia e Estatística; 2010 [citado 2018 mai 26]. Disponível em: https:// ww2.ibge.gov.br/home/estatistica/populacao/ censo2010/resultados_gerais_amostra/resultados gerais_amostra_tab_uf_xls.shtm.

19 Victora CG, Wagstaff A, Schellenberg JA, Gwatkin D, Claeson M, Habicht JP. Applying an equity lens to child health and mortality: more of the same is not enough. Lancet. 2003 Jul;362(9379):233-41.

20 Dias JCP. Human Chagas disease and migration in the context of globalization: some particular aspects. J Trop Med. 2013;2013:789758.

21 Monteiro WM, Magalhães LKC, Sá ARN, Gomes ML, Toledo MJO, Borges L, et al. Trypanosoma cruzi IV causing outbreaks of acute Chagas disease and infections by different haplotypes in the western Brazilian Amazonia. PLoS One. 2012;7(7): e41284.

22 Simões MV, Romano MMD, Schmidt A, Martins KSM, Marin-Neto JA. Chagas disease cardiomyopathy. Int J Cardiovasc Sci. 2018;31 (2):173-89. 
23 Matos R. Periferias de grandes cidades e movimentos populacionais. Cad Metropol. 2005; 13:71-105.

24 Rassi A, Rassi Jr A. Doença de Chagas aguda. In: Lopes AC, Guimarães HP, Lopes RD, Vendrame LS. Programa de atualização em medicina de urgência e emergência. Porto Alegre: Artmed; 2013. p. 41-85.

25 Sanmartino M, Crocco L. Conocimientos sobre la enfermedad de Chagas y factores de riesgo en comunidades epidemiológicamente diferentes de Argentina. Rev Panam Salud Publica. 2000;7(3): 173-8.

26 Ministério da Saúde (BR). Municípios de residência de casos agudos confirmados no SINAN no período de 2007 a 2016* [Internet]. Brasília: Ministério da Saúde; 2017 [citado 2017 dez 10]. Disponível em: https://www. saude.gov.br/images/pdf/2017/novembro/13/ LISTA-DE-MUNICIPIOS-DE-RESID--NCIA-DE-CASO S-AGUDOS-CONFIMARDOS-NO-SINAN.pdf.
27 Pinto AYN, Valente SA, Valente VC, Ferreira Jr AG, Coura JR. Acute phase of Chagas disease in the Brazilian Amazon region: study of 233 cases from Pará, Amapá and Maranhão observed between 1988 and 2005. Rev Soc Bras Med Trop. 2008 Nov-Dec;4 1 (6):602-14.

28 Shikanai-Yasuda MA, Carvalho NB. Oral transmission of Chagas disease. Clin Infect Dis. 2012;54(6):845-52.

29 Monteiro WM, Barbosa MGV, Toledo MJO, Fé FA, Fé NF. Série de casos agudos de doença de Chagas atendidos num serviço terciário de Manaus, Estado do Amazonas, de 1980 a 2006. Rev Soc Bras Med Trop. 2010 mar-abr;43(2):207-10.

30 Araújo-Jorge TC, Castro SL, organizadoras. Doença de Chagas: manual para experimentação animal. Rio de Janeiro: Fiocruz; 2000. 368 p.

31 Steindel M, Dias JCP, Romanha AJ. Doença de Chagas, mal que ainda preocupa. Cienc Hoje. 2005;37(217):32-8. 